\title{
TLN-05220, TLN-05223, new Echinosporamicin-type antibiotics, and proposed revision of the structure of bravomicins*
}

\author{
Arjun H Banskota ${ }^{1}$, Mustapha Aouidate ${ }^{2}$, Dan Sørensen ${ }^{3}$, Ashraf Ibrahim ${ }^{2}$, Mahmood Piraee ${ }^{4}$, \\ Emmanuel Zazopoulos ${ }^{2}$, Anne-Marie Alarco ${ }^{2}$, Henriette Gourdeau ${ }^{2}$, Christophe Mellon ${ }^{2}$, \\ Chris M Farnet ${ }^{2}$, Pierre Falardeau ${ }^{2}$ and James B McAlpine ${ }^{2}$
}

The deposited strain of the hazimicin producer, Micromonospora echinospora ssp. challisensis NRRL 12255 has considerable biosynthetic capabilities as revealed by genome scanning. Among these is a locus containing both type I and type II PKS genes. The presumed products of this locus, TLN-05220 (1) and TLN-05223 (2), bear a core backbone composed of six fused rings starting with a 2-pyridone moiety. The structures were confirmed by conventional spectral analyses including MS, and 1D and 2D NMR experiments. Comparison of both the ${ }^{1} \mathrm{H}$ and ${ }^{13} \mathrm{C}$ NMR data of the newly isolated compound with those of echinosporamicin and bravomicin A led us to propose a revision of the structure of the latter to include a 2-pyridone instead of the pyran originally postulated. Both compounds ( 1 and 2 ) possessed strong antibacterial activity against a series of grampositive pathogens including several strains of methicillin-resistant Staphylococcus aureus and vancomycin-resistant Enterococci (VRE), and cytotoxic activities against several human tumor cell lines. The TLN compounds are the first of this group with reported anticancer activity.

The Journal of Antibiotics (2009) 62, 565-570; doi:10.1038/ja.2009.77; published online 14 August 2009

Keywords: antibacterial; anticancer; bravomicin; Micromonospora echinospora; polycyclic aromatic; TLN-05220; TLN-05223

\section{INTRODUCTION}

The genomics of secondary metabolite biosynthesis recently evolved to the point in which analysis of the genome of an organism can define its biosynthetic capabilities for secondary metabolites. A genome scanning technique, requiring minimal amount of sequencing, has been developed in our laboratories, and used with our DECIPHER technology to analyze the genomes of bacteria for their secondary metabolite biosynthetic genes, greatly reducing the amount of sequencing required to define this capability. ${ }^{1-4}$ This approach not only ascertains the biosynthetic potential of a producing organism, but it provides the scientist with a handle to detect, isolate and structurally define a specific metabolite. We have shown this approach in the isolation and structural determination of an antifungal agent, ECO02301 from Streptomyces aizunensis ${ }^{5}$ and three 5-alkenyl-3,3(2H)furanones from two different Streptomyces species. ${ }^{6}$ This genome scanning approach, which was applied to define the biosynthetic capabilities of Micromonospora echinospora ssp. challisensis NRRL 12255 (this strain was deposited (as SCC 1411) to support US Patent $4,440,751),{ }^{7}$ revealed 13 secondary metabolite biosynthetic loci. In our experience, this is an unusually high number to find in a Micro- monospora species and led to the prioritization of this strain for investigation. A preliminary DECIPHERIT analysis annotated these loci as encoding a terpenoid, five mixed PKS/NRPS, four NRPS and three PKS biosynthetic systems. In this article, we describe the use of the genome scanning technique ${ }^{8,9}$ to identify and isolate new polycyclic aromatic antibiotics (TLN-05220, 1 and TLN-05223, 2) from this strain, the former was previously referred to as ECO-3396. ${ }^{1,2}$ Both compounds (1 and 2 ) possessed strong antibacterial activity against broadly resistant, gram-positive pathogens, and cytotoxic activities against several human tumor cell lines.

\section{RESULTS AND DISCUSSION}

M. echinospora ssp. challisensis NRRL 12255 was obtained from the National Center for Agricultural Utilization Research (NCAUR) in Peoria, IL, USA and was grown in shaken flasks in a dozen different fermentation media designed for the production of secondary metabolites. The whole cultures were then extracted by equal volumes of $\mathrm{MeOH}$ and the resultant aqueous/MeOH extracts were subjected to HPLC/MS/UV analyses. A number of these extracts contained a compound with UV absorption $\lambda_{\max }$ at 248 and $508 \mathrm{~nm}$, as might

\footnotetext{
${ }^{1}$ Institute for Marine Biosciences, National Research Council, Halifax, Nova Scotia, Canada; ${ }^{2}$ Thallion Pharmaceuticals, Alexander-Fleming, Montréal, Quebec, Canada; ${ }^{3}$ Merck-Frosst Canada, Trans Canada Highway, Kirkland, Quebec, Canada and ${ }^{4}$ Biotica Technology, Chesterford Research Park, Cambridge, UK Correspondence: Dr JB McAlpine, 730 Benson Lane, Green Oaks, IL 60048, USA. E-mail: jmcalpine@thallion.com

*Part IV in a series on Genomic Analysis for the Discovery of Novel Secondary Metabolites. TLN-05220 was previously referred to as ECO-3396.

Received 30 April 2009; revised 9 July 2009; accepted 22 July 2009; published online 14 August 2009
} 
be expected for a type II polyketide. This compound had MS peaks at $\mathrm{m} / \mathrm{z} 738.1$ (in the positive mode) and 736.1 (in the negative mode). Larger scale $(20 \times 500 \mathrm{ml})$ fermentations of M. echinospora ssp. challisensis NRRL 12255 were carried out in the two media, which gave the best yields of this metabolite. On harvest, the EtOAc extract gave TLN05220 (1) and TLN-05223 (2) (Figure 1) by Sephadex LH-20 (SigmaAldrich Canada, Oakville, ON, Canada) column chromatography followed by reversed phase HPLC.

TLN-05220 (1) was isolated as a red amorphous solid with molecular formula $\mathrm{C}_{30} \mathrm{H}_{31} \mathrm{~N}_{3} \mathrm{O}_{13}$ calculated from the MS data $(\mathrm{m} / \mathrm{z}$ $738.15(\mathrm{M}+\mathrm{H})^{+}$and $\left.736.10(\mathrm{M}-\mathrm{H})^{-}\right)$. The ${ }^{1} \mathrm{H}$ NMR spectrum (Table 1) of 1 displayed five aromatic protons including two sets of ortho-coupling protons, one methoxy, two methine, three methylene and three C-methyl groups. The carbon NMR spectrum (Table 2) on the other hand had 38 carbon signals including 23 quaternary carbons in aromatic and carbonyl region ( $\delta$ 111.4-187.1), indicating the presence of a complex aromatic system. The UV absorption of TLN05220 (1) at $\lambda_{\max } 508 \mathrm{~nm}$ further suggested that the rest of the molecule should be constructed by an extended conjugated system.

In-depth analyses of the gCOSY together with heteronuclear single quantum coherence (gHSQC) and heteronuclear multiple bond coherence (gHMBC) spectra led to the identification of the piperazinone and 1-methyl propyl groups as two partial structures. Additional sets of proton and carbon signals of the piperazinone moiety were also observed in the NMR spectra and are ascribed to the presence of slowly interchangeable rotamers. The presence of five carbonyl carbons $(\delta 187.1(\times 2), 183.3,181.9$ and 159.0), two sets of ortho-coupled aromatic protons $(\delta 8.63$ and $8.39 ; 7.47$ and 7.44$)$ and a singlet aromatic proton signal $(\delta 6.73)$ strongly suggested the presence of a polycyclic aromatic with a core backbone composed of six fused rings, similar to the albofungins, ${ }^{10}$ simaomicins ${ }^{11}$ and echinosporamicin 3. ${ }^{12,13}$ The presence of a carbonyl carbon at $\delta 187.1$ (C-5) resembles the situation with echinosporamicin $\mathbf{3}$, suggesting the presence of p-benzoquinone ring (ring E) instead of a $\gamma$-pyrone, as reported in the albofungin and simaomicin antibiotics, in which it could be derived through the oxidative cleavage of an echinospor- amicin-like ring E. Furthermore, the proton and carbon NMR data of TLN-05220 (1) were very similar to that of echinosporamicin 3, isolated from M. echinospora ssp. echinospora LL-P175. The only difference is the substitution patterns of ring $\mathrm{A}$ and ring $\mathrm{E}$. The presence of the second set of the ortho-coupled aromatic protons at $\delta$ 7.47 and 7.44 and two hydroxyl protons at $\delta 13.19$ strongly suggested that the ring $\mathrm{F}$ should be 6,9-dihydroxy substituted. The attachment of the 1-methyl propyl group was placed at C-16 based on the HMBC correlations observed between $\mathrm{H}-12$ and $\mathrm{C}-16$. An additional broad methylene signal at $\delta 5.23$ having HMBC correlation with carbonyls at $\delta 165.9$ and 159.0 indicated the connection of the piperazinone to the $\mathrm{N}$-alkyl pyridinone of the polycyclic chain through an amide linkage. Finally, the position of the methoxy group was determined to be at C-11 based on the HMBC correlation between the methoxyl protons at $\delta 4.04$ and $\mathrm{C}-11$ at $\delta$ 153.2. Accordingly, the planar structure of TLN-05220 was determined as $\mathbf{1}$. On the basis of the coupling constant of the protons on piperazinone moiety and the presence of similar rotamer signals as described for echinosporamicin, we are led to postulate a similar stereo-structure for these molecules. The stereochemistry of the 1-methyl propyl group remains undetermined.

An examination of related compounds in the literature brought our attention to the bravomicins, exemplified by the parent, bravomicin A, 4. ${ }^{14}$ The spectral data in this patent suggested a strong similarity between the structures of TLN-05220, echinosporamicin and bravomicin A, although these spectral data require some reinterpretation as the data given in the table do not mess with the spectra presented in the figures. Unfortunately, a sample of bravomicin A was not available to us to allow a thorough 2D NMR analysis and direct comparison of spectra measured under identical conditions. In the proton spectra (Table 1), the US patent lists the protons on C-17 at $\delta 1.31$ as a doublet and those on C-29 at $\delta 0.95$ as a triplet, whereas the Figure 3 in the patent indicates that the coupling patterns are reversed. Moreover, the triplet should clearly be assigned to the C-19 protons. (Note that the numbering system used in this article is that in Figure 1 and not the one given in the US patent.) The CMR table in the US patent does not include a carbon at $\sim \delta 45$; however, such a carbon is
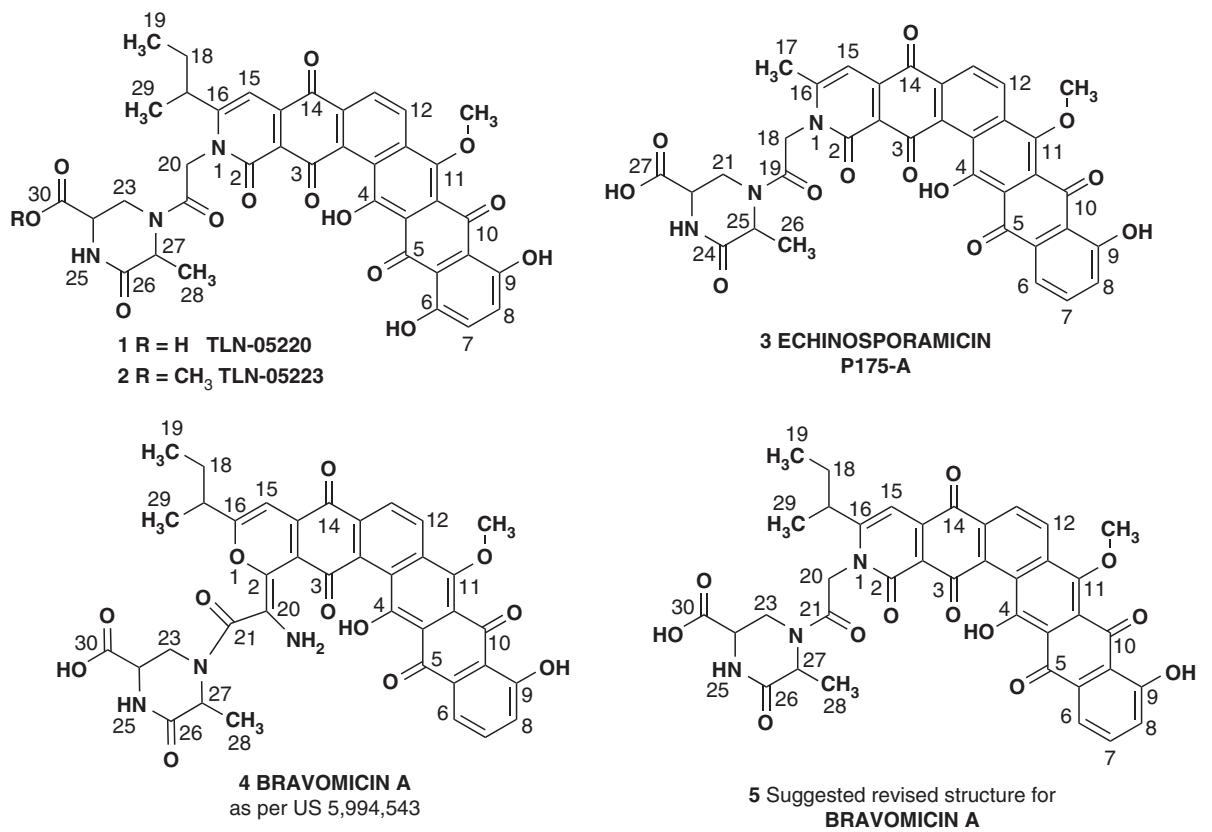

Figure 1 Structures. 
Table 1 The ${ }^{1} \mathrm{H}$ NMR data of TLN-05220 (1), TLN-05223 (2), Echinosporamicin (3) and Bravomicin (4)

\begin{tabular}{|c|c|c|c|c|c|}
\hline & Echinosporamicin (3) & $T L N-05220(1)^{a}$ & $T L N-05223(2)^{a}$ & Bravomicin (4) ${ }^{\mathrm{a}}$ & Revised bravomicin (4) \\
\hline $4-\mathrm{OH}$ & 15.37 & 14.64 & 14.64 & 15.38 & 15.38 \\
\hline 6 & 7.77 & - & - & 7.64 & 7.64 \\
\hline 7 & 7.75 & 7.47 & 7.19 & 7.69 & 7.69 \\
\hline 8 & 7.35 & 7.44 & 7.12 & 7.29 & 7.29 \\
\hline $9-\mathrm{OH}$ & 12.90 & 13.19 & 13.28 & 12.83 & 12.83 \\
\hline 15 & 6.75 & 6.73 & 6.69 & 6.72 & 6.72 \\
\hline 17 & $2.42,(2.39)$ & 2.67 & 2.62 & 2.68 & 2.68 \\
\hline $18^{b}$ & & $1.74,1.64$ & $1.74,1.62$ & $1.70,1.80$ & $1.70,1.80$ \\
\hline $19^{b}$ & & 0.91 & 0.90 & 0.95 & 0.95 \\
\hline $18,20^{\mathrm{b}}$ & $5.10(5.20)$ & $5.12(5.23)$ & $5.09(\mathrm{br})$ & - & $5.10(\mathrm{br})$ \\
\hline $21,23^{b}$ & $H_{a x}: 3.39(4.69)$ & $\mathrm{H}_{\mathrm{ax}}: 3.38(3.81)$ & $H_{a x}: 3.42(3.84)$ & $H_{a x}: 3.42(3.84)$ & $\mathrm{H}_{\mathrm{ax}}: 3.42(3.84)$ \\
\hline $26,28^{b}$ & $1.57(1.31)$ & $1.60(1.32)$ & $1.60(1.32)$ & $1.57(1.31)$ & $1.57(1.31)$ \\
\hline $29^{b}$ & - & 1.27 & 1.24 & 1.31 & 1.31 \\
\hline $30-\mathrm{OCH}_{3}{ }^{\mathrm{b}}$ & - & - & 3.61 & - & - \\
\hline $11-\mathrm{OCH}_{3}$ & $4.00(\mathrm{~s})$ & 4.04 & 3.86 & $3.97(\mathrm{~s})$ & 3.97 \\
\hline
\end{tabular}

aChemical shifts on the parentheses belong to the minor rotamer.

${ }^{b}$ Carbon numbers for TLN-05220 (1), TLN-05223 (2) and Bravomicin (4) 17- $\mathrm{NH}_{2}$ in the patent structure of Bravomicin is assigned as $\delta 5.10$.

clearly evident in Figure 4 of the patent. The corresponding carbon, the attached protons at $\sim \delta 5.1$ and their HMBC correlations have a critical role in the attachment of the piperazinone moiety in the structural determinations of both TLN-05220 and echinosporamicin. Clearly the data for the revised structure of bravomicin A (6-deoxy TLN-05220) cannot be regarded as rigorous; however, it is highly suggestive.

TLN-05223 (2) was also isolated as a red amorphous solid with molecular formula $\mathrm{C}_{39} \mathrm{H}_{33} \mathrm{~N}_{3} \mathrm{O}_{13}$ calculated from the MS data $\left(\mathrm{m} / z 752.6(\mathrm{M}+\mathrm{H})^{+}\right.$and $\left.750.4(\mathrm{M}-\mathrm{H})^{-}\right)$. The ${ }^{1} \mathrm{H}$ and ${ }^{13} \mathrm{C}$ NMR signals of 2 were identical to that of 1 except the presence of an additional $O$-methyl group $(\delta 3.61)$. The identical mass fragment of both 1 and 2 at $m / z 580$, that is, $\left(\mathrm{M}+\mathrm{H}-\right.$ piperazinone moiety) ${ }^{+}$ suggested that $\mathbf{2}$ is the methyl ester of $\mathbf{1}$, and may possibly be an artifact of the isolation procedure.

One of the 13 biosynthetic loci determined using the DECIPHER genomic analysis platform was ascribed to the biosynthesis of TLN05220 and TLN-05223. This locus (the DNA and protein sequences that comprise the TLN-05220 gene cluster are deposited in GenBank under accession number FJ915123) spanned approximately $65 \mathrm{~Kb}$ and contained a small PKS type I gene in which the adenylation (AT) domains each contained the YASH motif specifying the incorporation of methylmalonate. This PKS type I gene is assumed to result in carbons 16 through 19 and 29, and was slightly upstream of the PKS type II genes that would give rise to the hexacyclic system. Moreover, in the vicinity were a number of genes responsible for oxidative and cyclization functions, and an $O$-methyl transferase. Surprisingly, there were no genes in this locus as sequenced, nor in any of the other 12 loci detected, which would be annotated as NRPS genes encoding a glycine, an alanine and one other (possibly serine) amino acid residue that would be expected to give rise to the glycyl piperazinone moiety, as defined by carbons 20 through 28 and 30 . As extensive sequencing analysis was performed on both extremities of this locus, it is possible that the NRPS genes presumed to be involved in the biosynthesis of glycyl piperazinone moiety of compounds $\mathbf{1}$ and $\mathbf{2}$ are provided in trans by a yet undefined region of the genome.

The compounds were tested for their antibacterial activity against seven pathogenic strains. The MICs are summarized in Table 3. Both compounds (1 and 2 ) possessed significant antibacterial activity against all strains tested. The MIC values were comparable to those of vancomycin, which was used as a control. The potency of the polycyclic aromatics was stronger than that of vancomycin against all tested pathogens except Streptococcus pneumoniae PenR (LSPQ 3349). Although the specific strains were different, the activity of TLN-05220 seems to be similar to that reported for echinosporamicin ${ }^{12,13}$ and bravomicin A. ${ }^{14}$

Compound 1 was also evaluated for antitumor activity. Bravomicin and echinosporamicin were reported as gram-positive antibacterial agents, the latter specifically described as having no significant anticancer activity against HCT-116 ${ }^{13}$. As summarized in Table 4, TLN05220 had potent growth inhibitory activity (sub-micromolar range) on the four human solid tumor cell lines examined. The compound was less active on K-562, an erythroleukemia cell line derived from a chronic myeloid leukemia patient in blast crisis.

As the prostate cell line was the most sensitive, the in vivo antitumor activity of TLN-05220 was evaluated in nude mice bearing human prostate (PC3) tumors (Figure 2). Animals from the vehicle control group were killed when the tumor volume reached $\sim 1000 \mathrm{~mm}^{3}$. Out of 10 mice used in the control group, 1 mouse reached the end point by day 26, 7 mice reached the end point by day 40 and the remaining 3 animals on day 43 . At $25 \mathrm{mg} \mathrm{kg}^{-1}$ on alternate days, i.p. administration of TLN-05220 caused severe abdominal trauma by day 7 and a death on day 13. In total, seven more deaths occurred between days 27 and 50. On day 36 , the median tumor volume for this group (six mice) was $467 \mathrm{~mm}^{3}$. The median tumor volume for the vehicle-treated group (nine mice) was $615 \mathrm{~mm}^{3}$. This reflects a tumor reduction of $24 \%$ and is not statistically significant. An i.v. administration of TLN-05220 at 
Table 2 CMR assignments

\begin{tabular}{|c|c|c|c|c|}
\hline Carbon $^{\mathrm{a}}$ & Echinosporamicin & $T L N-05220$ & Bravomicin & Revised bravomicin \\
\hline 2 & $157.7,157.7$ & 159.0, 159.1 & $164.3,164.3$ & $157.7,157.7$ \\
\hline $2 a$ & $119.8,119.9$ & $121.4,121.4$ & $120.0,120.0$ & $120.0,120.0$ \\
\hline 3 & $181.9,181.9$ & $181.9,181.9$ & 180.7. 180.7 & 180.7. 180.7 \\
\hline $3 a$ & $137.4,137.4$ & $138.6,138.6$ & $141.6,141.6$ & $141.6,141.6$ \\
\hline $3 b$ & $124.6,124.6$ & $127.9,127.9$ & $124.3,124.3$ & $124.3,124.3$ \\
\hline 4 & $161.0,161.0$ & $165.2,165.2$ & $161.0,161.0$ & $161.0,161.0$ \\
\hline $4 a$ & 110.1110 .1 & $111.4,111.4$ & $109.9,109.9$ & $109.9,109.9$ \\
\hline 5 & $187.2,187.2$ & $187.1,187.1$ & $187.0,187.0$ & $187.0,187.0$ \\
\hline $5 a$ & $132.7,132.7$ & $114.6,114,6$ & $132.6,132.6$ & $132.6,132.6$ \\
\hline 6 & $118.5,118.5$ & $157.1,157.1$ & $118.4,118.4$ & $118.4,118.4$ \\
\hline 7 & $136.9,136.9$ & $130.2,130.2$ & $136.9,136.9$ & $136.9,136.9$ \\
\hline 8 & $124.4,124.4$ & $130.2,130.2$ & $124.7,124.7$ & $124.7,124.7$ \\
\hline 9 & $161.8,161.8$ & $157.9,157.9$ & $161.8,161.8$ & $161.8,161.8$ \\
\hline $9 a$ & $117.3,117.3$ & $116.1,116.1$ & $117.2,117.2$ & $117.2,117.2$ \\
\hline 10 & $186.5,186.5$ & 187.1, 187.1, & $186.3,186.3$ & $186.3,186.3$ \\
\hline $10 a$ & $120.1,120.1$ & $122.0,122.0$ & $119.9,119.9$ & $119.9,119.9$ \\
\hline 11 & $151.8,151.8$ & $153.2,153.2$ & $151.9,151.9$ & $151.9,151.9$ \\
\hline $11 a$ & $138.5,138.5$ & $140.2,140.2$ & $137.5,137.5$ & $137.5,137.5$ \\
\hline 12 & $128.4,128.4$ & $129.8,129.8$ & $128.5,128.5$ & $128.5,128.5$ \\
\hline 13 & $126.8,126.8$ & $128,3,128.3$ & $126.8,126.8$ & $126 / 8,126.8$ \\
\hline $13 a$ & $133.8,133.8$ & $134.9,134.9$ & $133.8,133.8$ & $133.8,133.8$ \\
\hline 14 & $180.8,180.8$ & $183.3,183.3$ & $182.0,182.0$ & $182.0,182.0$ \\
\hline $14 a$ & $141.6,141.5$ & $143.0,143.0$ & $138.5,138.5$ & $138.5,138.5$ \\
\hline 15 & $100.5,100.5$ & $98.4,98.4$ & $97.0,97.0$ & $97.0,97.0$ \\
\hline 16 & $155.4,155.6$ & $165.3,165.3$ & $163.9,163.9$ & $163.9,163.9$ \\
\hline 17 & $21.0,21.2$ & $39.6,39.6$ & $20.0,20.0$ & $38.1,38.1$ \\
\hline $18^{\mathrm{b}}$ & & $30.1,30.1$ & $38.1,38.1$ & $28.3,28.3$ \\
\hline $19^{b}$ & & $13.1,13.1$ & $28.3,28.3$ & $11.8,11.8$ \\
\hline $18,20^{\mathrm{b}}$ & $45.7,45.6$ & $45.9,45.9$ & $157.7,157.7$ & $\sim 45^{a}$ \\
\hline $19,21^{b}$ & $164.4,163.9$ & $165.9,165.5$ & $164.7,164.7$ & $164.7,164.3$ \\
\hline $21,23^{b}$ & $37.3,40.4$ & $38.6,42.1$ & $37.1,40.3$ & $37.1,40.3$ \\
\hline $22,24^{b}$ & $52.5,53.1$ & $54.1,54.6$ & $52.6,53.2$ & $52.6,53.2$ \\
\hline $24,26^{b}$ & $168.5,168.8$ & $169.9,170.1$ & $168.8,168.8$ & $168.8,168.8$ \\
\hline $25,27^{b}$ & $52.7,51.0$ & $54.1,52.5$ & $52.7,51.1$ & $52.7,51.1$ \\
\hline $26,28^{b}$ & $17.5,16.0$ & $19.0,17.5$ & $17.5,16.0$ & $17.5,16.0$ \\
\hline $29^{b}$ & & $21.6,21.6$ & $11.8,11.8$ & $20.0,20.0$ \\
\hline $27,30^{\mathrm{b}}$ & $172.3,172.3$ & $173.5,173.3$ & $172.4,172.2$ & $172.4,172.2$ \\
\hline $\mathrm{OCH}_{3}$ & $62.7,62.7$ & $64.4,64.4$ & $62.7,62.7$ & $62.7,62.7$ \\
\hline
\end{tabular}

aln the patent, this peak does not appear in the assigned peaks in Table 2; however, it is clearly evident in Figure 4, the CMR spectrum of bravomicin A.

bThe difference in structures requires different numbering for structurally comparable carbons.

Table 3 Antibacterial activity of compound 1 and 2; MICs are expressed in $\mu \mathrm{g} \mathrm{ml}^{-1}$

\begin{tabular}{lccc} 
Strain & TLN-05220 (1) & TLN-05223 (2) & Vancomycin \\
\hline S. aureus ATCC 6538P & 0.016 & 0.0625 & 1 \\
S. aureus MRS3 ATCC 700699 & 0.016 & 0.125 & 4 \\
S. pneumoniae LSPQ13412 & $0.5-1$ & 1 & 0.5 \\
S. pneumoniae PenR LSPQ 3349 & $4-16$ & 1 & 0.5 \\
E. faecalis VRE-1 ATCC 29212 & 0.063 & 1 & $4-8$ \\
E. faecalis VRE-2 ATCC 51299 & 0.125 & 1 & 32 \\
Clostridium difficile ATCC 9689 & 0.06 & 2 & 0.5 \\
\hline
\end{tabular}

$10 \mathrm{mg} \mathrm{kg}^{-1}$ caused severe toxicity to the tail vein, necessitating the i.p. route for subsequent dosing. The median tumor volume for this group on day 36 (eight mice-two animals had to be killed due to their tumors reaching $1000 \mathrm{~mm}^{3}$ ) was $634 \mathrm{~mm}^{3}$, and thus not significantly different from the vehicle control group. In summary, although TLN05220 was potent in inhibiting the growth of PC3 cells, it failed to produce in vivo antitumor efficacy. It was felt that the compound had a narrow therapeutic index and also that the pharmacokinetics of the drug were not suitable for i.v. or i.p. dosing.

\section{EXPERIMENTAL SECTION}

\section{General}

Optical rotations were measured at $589 \mathrm{~nm}$ in a 1-dm cell on a Perkin Elmer polarimeter Model 241 (Perkin Elmer, Waltham, MA, USA). The NMR spectra were measured on a Varian Unity Inova $500 \mathrm{MHz}$ spectrophotometer (Varian, Palo Alto, CA, USA) with dimethylsulfoxide- $d_{6}$ solution and are referenced to TMS. The analytical HPLC was carried out with a Waters Alliance 2690 instrument (Waters, Milford, MA, USA) equipped with Micromass ZQ electrospray (Waters) source and Waters 996 diode array UV detectors (Waters). Semi-preparative HPLCs were performed on Waters 600 Controller instrument (Waters) with Waters 996 diode array UV detector (Waters). All the chemicals and solvents used for the purifications were HPLC grade.

\section{Genome scanning}

The genome of M. echinospora ssp. challisensis NRRL 12255 was analyzed by the genome scanning technique as described previously by Zazopoulos et al. ${ }^{3,4}$ and the nucleotide and protein sequenced were deposited in GenBank (accession number FJ915123).

\section{Fermentation}

M. echinospora ssp. challisensis NRRL 12255, which was obtained from the NCAUR in Peoria, IL, USA, was maintained and sporulated on agar plates of ATCC culture medium 172 (glucose $10 \mathrm{gl}^{-1}$, soluble starch $20 \mathrm{gl}^{-1}$, yeast extract $5 \mathrm{gl}^{-1}, \mathrm{~N}$-Z-amine $\mathrm{A} 5 \mathrm{gl}^{-1}, \mathrm{CaCO}_{3} 19 \mathrm{gl}^{-1}$ and agar $15 \mathrm{gl}^{-1}$ in distilled water). The surface growth from the agar plate was homogenized and transferred to a $125 \mathrm{ml}$ flask containing three glass beads ( $5 \mathrm{~mm}$ diameter), and $25 \mathrm{ml}$ of sterile FBB medium (potato dextrin $24 \mathrm{gl}^{-1}$, beef extract $3 \mathrm{gl}^{-1}$, Bacto Casitone $5 \mathrm{gl}^{-1}$, glucose $5 \mathrm{gl}^{-1}$, yeast extract $5 \mathrm{gl}^{-1}$ and $\mathrm{CaCO}_{3} 4 \mathrm{gl}^{-1}$ in distilled water). The $\mathrm{pH}$ of the medium was adjusted at 7.0 before adding $\mathrm{CaCO}_{3}$ and then autoclaved. This vegetative culture was incubated at $28^{\circ} \mathrm{C}$ for about $70-72 \mathrm{~h}$ on a shaker with a $2.5 \mathrm{~cm}$ throw and set at 250 r.p.m.

The vegetative culture $(10 \mathrm{ml}$ aliquots) was used to inoculate $2-1$ baffled flasks each containing $500 \mathrm{ml}$ of sterile media; medium A (glucose $10 \mathrm{gl}^{-1}$, sucrose $20 \mathrm{gl}^{-1}$, cane molasses $20 \mathrm{gl}^{-1}$, Soytone-peptone $5 \mathrm{gl}^{-1}$ and $\mathrm{CaCO}_{3}$ $2.5 \mathrm{gl}^{-1}$ in distilled water) and medium $\mathrm{B}$ (maltose $4 \mathrm{gl}^{-1}$, yeast extract $4 \mathrm{gl}^{-1}$ and malt extract $10 \mathrm{gl}^{-1}$ in distilled water). Both media were adjusted at $\mathrm{pH} 7.0$ before sterilization. The fermentation batches were incubated aerobically on a shaker (200 r.p.m.) with a $2.5 \mathrm{~cm}$ throw at $28^{\circ} \mathrm{C}$ for a period of 4 days.

\section{Extraction and isolation}

Method A. The whole fermentation broth of the culture medium A $(20 \times 500 \mathrm{ml})$ was adjusted to $\mathrm{pH} 3.5$ adding sulfuric acid and extracted with ethyl acetate $(2 \times 5 \mathrm{l})$. The organic phase was dried over anhydrous $\mathrm{MgSO}_{4}$ and concentrated under reduced pressure. The residue was then dissolved in methanol $(12 \mathrm{ml})$ and the $\mathrm{MeOH}$ soluble part was subjected to a Sephadex LH-20 column $(3.5 \times 100 \mathrm{~cm})$ eluted with $\mathrm{MeOH}$ under gravity. The fractions $(10 \mathrm{~min}$ per fraction) were collected after $2 \mathrm{~h}$ of sample injection. Fractions 17-21 were pooled and concentrated under reduced pressure, which gave a red precipitate. The red precipitate was collected by filtration to give pure TLN-05220 (1, $7.93 \mathrm{mg})$.

Method B. The fermentation broth of the culture medium B $(20 \times 500 \mathrm{ml})$ was adjusted to $\mathrm{pH} 3.5$ adding sulfuric acid and extracted with ethyl acetate $(2 \times 51)$. The organic phase was dried over anhydrous $\mathrm{MgSO}_{4}$ and evaporated under reduced pressure. The residue was dissolved in methanol/dimethyl sulfoxide (DMSO) (9:1, $20 \mathrm{ml}$ ) and subjected to a Sephadex LH-20 column $(3.5 \times 100 \mathrm{~cm})$ eluted with $\mathrm{MeOH}$ under gravity. The fractions $(10 \mathrm{~min}$ per fraction) were collected after $2 \mathrm{~h}$ of sample injection. Fractions $2-10$ were pooled and concentrated under reduced pressure. The residue was then dissolved in $\mathrm{DMSO} / \mathrm{MeOH}$ mixture $(3: 1,5 \mathrm{ml})$ and subjected to HPLC purification after filtering through a $0.45 \mu \mathrm{m} 13-\mathrm{mm}$ Acrodisc GHP syringe 
filter (Waters). The HPLC was performed on a Waters 600 Controller instrument (Waters) using a Waters NovaPak C18 column (Waters) $(5 \mu \mathrm{m}$, $25 \times 550 \mathrm{~mm}$ ), and a gradient of $0.01 \%$ aqueous acetic acid/acetonitrile $\left(20 \mathrm{ml} \mathrm{min}^{-1}, 80: 20\right.$ to $20: 80(0-15 \mathrm{~min}) ; 20: 80$ to $\left.0: 100(15-20 \mathrm{~min})\right)$. TLN$05220(1,8.63)$ and TLN-05223 (2, $0.5 \mathrm{mg})$ were obtained from multiple injections, eluting at 14.5 and $17.1 \mathrm{~min}$, respectively.

1: $[\alpha]^{20}{ }_{\mathrm{D}}-60^{\circ}(\sim 0.001 \mathrm{MeOH}) \mathrm{UV}\left(\lambda_{\max }\right) 249,280(\mathrm{sh}), 335$ (sh) and $508 \mathrm{~nm}$; MS (ESI in positive mode) $\mathrm{m} / z 760.0(\mathrm{M}+\mathrm{Na})^{+}, 738.1(\mathrm{M}+\mathrm{H})^{+}, 579.8$ $(\mathrm{M}+)^{+}$; MS (ESI in negative mode) $\mathrm{m} / z 736.1(\mathrm{M}-\mathrm{H})^{-}$; HRMS 738.1939 calcd for $\mathrm{C}_{38} \mathrm{H}_{31} \mathrm{~N}_{3} \mathrm{O}_{13}(\mathrm{M}+\mathrm{H})^{+} 738.1935 \Delta=0.57$ p.p.m. The ${ }^{1} \mathrm{H}$ and ${ }^{13} \mathrm{C}$ NMR data are in Table 1 and 2, respectively.

2: UV $\left(\lambda_{\max }\right) 249,280(\mathrm{sh}), 335(\mathrm{sh})$ and $508 \mathrm{~nm}$; MS (ESI in positive mode) $m / z 774.6(\mathrm{M}+\mathrm{Na})+, 752.6(\mathrm{M}+\mathrm{H})^{+}, 580.4(\mathrm{M}+)^{+}$; MS (ESI in negative mode) $m / z 750.4(\mathrm{M}-\mathrm{H})^{-}$; HRMS 752.2083 calcd for $\mathrm{C}_{39} \mathrm{H}_{33} \mathrm{~N}_{3} \mathrm{O}_{13}(\mathrm{M}+\mathrm{H})^{+}$ $752.2086 \Delta=0.40$ p.p.m. The ${ }^{1} \mathrm{H}$ and ${ }^{13} \mathrm{C}$ NMR data are in Table 1 and 2, respectively.

\section{Antibacterial activity}

Antibacterial activity of the isolated compounds was measured by determining the MIC against seven pathogenic strains, namely Staphylococcus aureus (ATCC 6538P), S. aureus MRS3 (ATCC 700699), S. pneumoniae (LSPQ 13412), S. pneumoniae PenR (LSPQ 3349), Enterococcus faecalis VRE-1 (ATCC 29212), E. faecalis VRE-2 (ATCC 51299) and Clostridium difficile (ATCC 9689). The antibacterial experiments were performed according to the National Committee for Clinical Laboratory Standards (NCCLS) guideline M7-A5. ${ }^{15}$

Stock solutions of the tested compounds were prepared in DMSO $(100 \times)$ and diluted with Mueller-Hinton test medium as twofold series over 11 points from 64 to $0.06 \mu \mathrm{g} \mathrm{ml}^{-1}$. An aliquot of each stock solution was diluted 50 -fold in test medium described below to give a set of eleven $2 \times$ solutions. In total, $50 \mu \mathrm{l}$ of each of the eleven $2 \times$ solutions were aliquoted into the corresponding wells of a 12-well row microtiter plate, with the final well reserved for a medium-alone control. Vancomycin (Sigma-Aldrich Canada), used as positive control, was prepared as $2 \times$ stock solutions in Mueller-Hinton test medium

Table 4 Effect of TLN-05220 on cell growth

\begin{tabular}{llcc}
\hline & & \multicolumn{2}{c}{$T L N-05220 \mathrm{Gl}_{50}(\mu \mathrm{M})$} \\
\cline { 3 - 4 } Cell lines & Tissue origin & EXP 1 & EXP 2 \\
\hline MCF7 & Breast & 0.59 & 0.22 \\
HCT 116 & Colon & 0.09 & 0.71 \\
PC-3 & Prostate & $<0.01$ & 0.04 \\
NCI-H460 & Lung & 0.28 & 1.22 \\
K-562 & Leukemia & 4.62 & 9.7 \\
\hline
\end{tabular}

ranging from 64 to $0.06 \mu \mathrm{g} \mathrm{ml}^{-1}$ (a twofold dilution series over 11 points). An aliquot of $50 \mu \mathrm{l}$ of each concentration (at $2 \times$ ) was then transferred to 96-well microtiter plate to obtain a series of eleven twofold dilutions.

An isolated colony of each of the seven indicator strains was used to inoculate tubes containing $2 \mathrm{ml}$ of test medium. Mueller-Hinton test medium was used for S. aureus (ATCC 6538P), S. aureus MRS3 (ATCC 700699), S. pneumoniae (LSPQ 13412), S. pneumoniae PenR (LSPQ 3349), C. difficile (ATCC 9689) and Brain Heart Infusion test medium was used for E. faecalis VRE-1 (ATCC 29212) and E. faecalis VRE-2 (ATCC 51299) indicator strains. Cells were grown overnight at $35^{\circ} \mathrm{C}$ with shaking. Inoculum density for each indicator strain was adjusted to $\mathrm{OD}_{600}=0.1$ in $5 \mathrm{ml} 0.85 \%$ saline, then further diluted 1/100 in appropriate medium. In total, $50 \mu \mathrm{l}$ of the final dilution (in test medium) of each indicator strain was added to each well of a 12-well row. This brings the final dilution of the test compound or control compound in solution to $1 \times$. The final inoculum has approximately $5 \times 10^{5} \mathrm{CFU} \mathrm{ml}^{-1}$.

The indicator strains were incubated with 11 concentrations of each of test compounds, vancomycin (Sigma) control and one medium-alone control. For MIC determination, assay plates were incubated at $35^{\circ} \mathrm{C}$ for $16-20 \mathrm{~h}$. The MIC for each indicator was assessed as the lowest concentration of the test compound resulting in total absence of growth and is shown in Table 3.

\section{Antitumor activity: in vitro studies}

Human breast (MCF7), colon (HCT116), prostate (PC3), lung (NCI-H460) and leukemia (K562) tumor cell lines were purchased from the American Type Culture Collection (Manassas, VA, USA). Cells were grown in RPMI 1640 supplemented with $2 \mathrm{~mm}$ L-glutamine and $10 \%$ heat-inactivated fetal bovine serum (FBS; Cambrex, Verviers, Belgium) and maintained in a humidified atmosphere at $37^{\circ} \mathrm{C}$ with $5 \% \mathrm{CO}_{2}$. Exponentially growing tumor cells $(5000$ 10000 cells per well depending on their doubling time; cell number was determined with a hemocytometer) were seeded in 96-well flat-bottom microtiter plates. The following day, cells were exposed to six different concentrations of TLN-05220 $(20,10,5,1,0.1$ and $0.01 \mu \mathrm{M})$ in a final volume of $200 \mu \mathrm{l}$ of RPMI $10 \%$ FBS and $0.1 \%$ DMSO for $96 \mathrm{~h}$. The in vitro cytotoxic activity was determined by a standard 3-(4,5-Dimethylthiazol-2-yl)-2,5-diphenyltetrazolium bromide (MTT) assay. ${ }^{16}$ All measurements were done in quadruplicate and each experiment was performed twice. A control plate, in which MTT was added to the cells after the overnight incubation period before compound addition, was used to determine day 1 absorbance reading $(0 \mathrm{~h})$. The dose response inhibition of proliferation (GI) for each concentration was determined following the formula:

$$
\mathrm{GI}=\frac{\mathrm{OD}_{\text {drug-exposed wells }(96 \mathrm{~h})}-\mathrm{OD}_{\text {drug-free wells }(0 \mathrm{~h})}}{\mathrm{OD}_{\text {drug-free wells }(96 \mathrm{~h})}-\mathrm{OD}_{\text {drug-free wells }(0 \mathrm{~h})}}
$$

The $\mathrm{GI}_{50}$ was estimated from individual inhibition curves and represents the concentration of drug that inhibits $50 \%$ of the cell growth as compared with

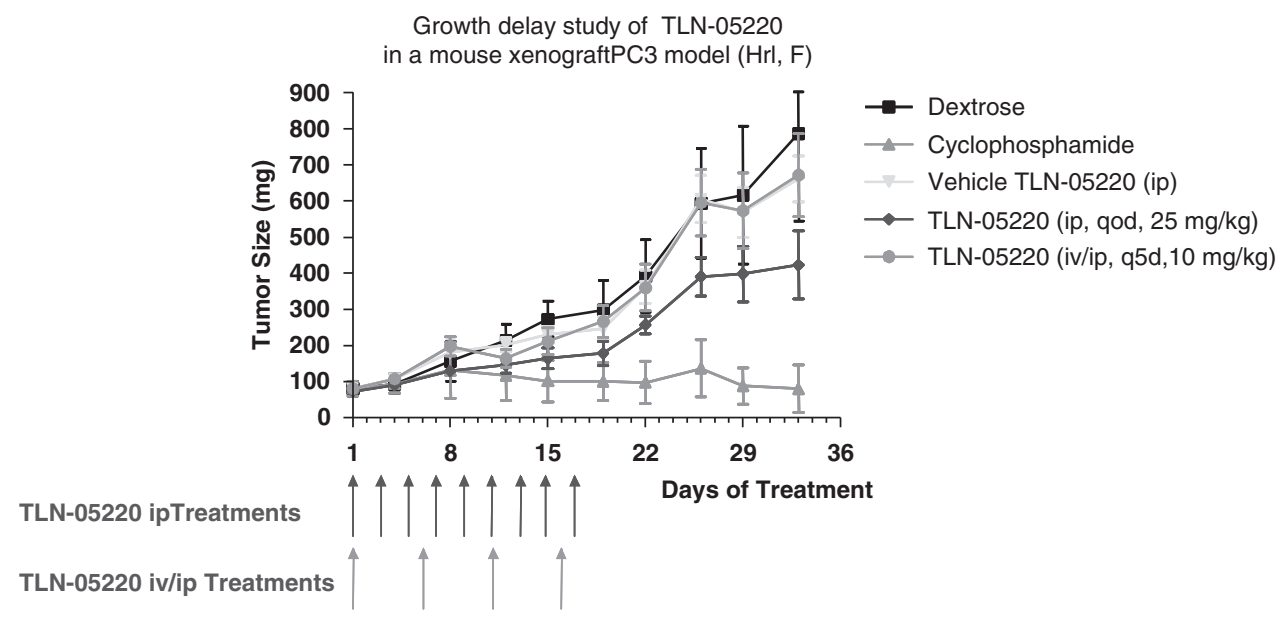

Figure 2 Growth delay study of TLN-05220 in a mouse xenograft PC3 model (Hrl, F). 
non-treated control cells (calculated with the PrismPad computer program (GraphPad software, San Diego, CA, USA)).

\section{In vivo studies}

The human PC-3 prostate carcinoma line used for these studies was maintained in athymic nude mice by serial engraftment. The study was performed at Piedmont Research Center (Morrisville, NC, USA), which is accredited by AAALAC International. A tumor fragment $\left(1 \mathrm{~mm}^{3}\right)$ was implanted SC into the right flank of male Nude-Foxn1nu/Foxn1+ mice (8-9 weeks old; Harlan, Indianapolis, IN, USA). When tumor volumes reached $80-120 \mathrm{~mm}^{3}$, mice were randomized ( 10 mice per group) and treatment began (day 1 of study $=19$ days after tumor fragment inoculation). Group 1 received drug-free vehicle (5\% ethanol, $4.5 \%$ glycocholate, $6 \%$ ethanolic polysorbate 80 and $84.5 \%$ dextrose-5\%), i.p., once daily on alternate days for nine doses (qod $\times 9$ ). Group 2 (positive control group), received cyclophosphamide i.p. at $100 \mathrm{mg} \mathrm{kg}^{-1}$, once daily on days $1-5$ ( $\mathrm{qd} \times 5$ ). Group 3 received TLN-05220 i.p. at $25 \mathrm{mg} \mathrm{kg}^{-1}$, once daily on alternate days for nine doses (qod $\times 9$ ). Group 4 received TLN-05220 i.v. (first dose) and i.p. (remaining three doses) at $10 \mathrm{mg} \mathrm{kg}^{-1}$, on days $1,6,11$ and $16(\mathrm{q} 5 \mathrm{~d} \times 4)$. Tumor growth was followed every other day by measuring tumor length $(L)$ and width $(W)$ using a caliper. Measurements were converted to tumor volumes (TV; $\mathrm{mm}^{3}$ ) using the standard formula, $\mathrm{TV}=\left(L \times W^{2}\right) / 2$.

\section{ACKNOWLEDGEMENTS}

We thank Professor André B. Charette of the Université de Montréal for the use of his polarimeter.

1 Alarco, A. M., Banskota, A., McAlpine, J., Farnet, C., Falardeau, P. \& Zazopoulos, E. Profiling of new antitumor natural products discovered using a genomics/cheminformatics-based drug discovery platform. Proc. Am. Assoc. Cancer Res. 47, A1992 (2006)
2 Gross, H. Genome mining-a concept for the discovery of new natural products. Curr. Opin. Drug Discov. Devel. 12, 207-219 (2009).

3 Zazopoulos, E. et al. A genomics-guided approach for discovering and expressing cryptic metabolic pathways. Nat. Biotechnol. 21, 187-190 (2003).

4 Zazopoulos, E. \& Farnet, C. M. Improving drug discovery from microorganisms. Natural Products: Drug Discovery and Therapeutic Medicine (eds Zhang, L. and Demain, A. L.) 95-106 (Humana Press Inc., Totowa, NJ, USA, 2005).

5 McAlpine, J. B. et al. Microbial genomics as a guide to drug discovery and structural elucidation: ECO-02301, a novel antifungal agent, as an example. J. Nat. Prod. 68, 493-496 (2005).

6 Banskota, A. H. et al. Isolation and identification of three new 5-Alkenyl-3,3(2H)furanones from two Streptomyces species using a genomic screening approach. J. Antibiot. 59, 168-176 (2006).

7 Waitz, J. A., Marquez, J. A., Patel, M. G. \& Horan, A. C. Broad spectrum antibiotic complex produced by a novel micromonospora. US 4,440,751, April 3 (2001).

8 Sørensen, D., McAlpine, J. B., Piraee, M., Farnet, C. M. \& Zazopoulos, E. Genome scanning technology reveals an antibacterial compound (ECO-0501) of a new structural class from the vancomycin-producer Amycolatopsis orientalis 44th ICAAC: no. F-720a, Washington, DC (2004).

9 McAlpine, J. B. et al. The power of genomic analysis in the discovery of novel secondary metabolites 46th Annual Meeting of American Society of Pharmacognosy: no. 0-21, Corvallis, OR, USA (2005).

10 Gurevich, A. I. et al. The structure of albofungin. Tet. Lett. 13, 1751-1754 (1972).

11 Carter, G. T., Goodman, J. J., Torrey, M. J., Borders, D. B. \& Gould, S. J. Biosynthetic origin of the carbon skeleton of sisaomicin A, a hexacyclic xanthone antibiotic. J. Org. Chem. 54, 4321-4323 (1989).

12 He, H., Yang, H. Y., Luckman, S. W. \& Bernam, V. S. Antibiotic P175-A and semisynthetic derivatives thereof US Patent Application. US 2004/0220195 Nov. 4, (2004).

$13 \mathrm{He}, \mathrm{H}$. et al. Echinosporamicin, a new antibiotic produced by Micromonospora echinospora ssp. echinospora, LL-P175. Helv Chim. Acta 87, 1385-1391 (2004).

14 Shu, Y-Z., Chen, J., Lam, K. S., Veitch, J. A. \& Brown, D. Antibiotic Bravomicins US Patent 5, 994, 543, Nov. 30, (1999).

15 Methods for Dilution Antimicrobial Susceptibility Tests for Bacteria That Grow Aerobically; Approved Standard-Fifth Edition (NCCLS document M7-A5, ISBN 1-56238394-9 Pennsylvania, USA).

16 Plumb, J. A., Milroy, R. \& Kaye, S. B. Effects of the $\mathrm{pH}$ dependence of 3-(4,5dimethylthiazol-2-yl)-2,5-diphenyl-tetrazolium bromide-formazan absorption on chemosensitivity determined by a novel tetrazolium-based assay. Cancer Res. 49, 4435-4440 (1989). 